\title{
Discovery of Small Molecule Entry Inhibitors Targeting the Fusion Peptide of SARS-CoV-2 Spike Protein
}

Xin $\mathrm{Hu}^{1, *}$, Catherine Z. Chen ${ }^{1}$, Miao Xu $\mathrm{X}^{1}$, Zongyi $\mathrm{Hu}^{2}$, Hui Guo ${ }^{1}$, Zina Itkin ${ }^{1}$, Paul Shinn ${ }^{1}$, Parker Irvin ${ }^{2}$, Madeleine Leek ${ }^{2}$, T. Jake Liang ${ }^{2}$, Min Shen ${ }^{1}$, Wei Zheng ${ }^{1}$, Matthew D. Hall,*

${ }^{1}$ National Center for Advancing Translational Sciences, National Institutes of Health, 9800 Medical Center Drive, Rockville, MD, 20850, USA

${ }^{2}$ Liver Diseases Branch, National Institute of Diabetes and Digestive and Kidney Diseases, National Institutes of Health, 10 Center Drive, Bethesda, MD 20892, USA

\section{Supporting Information}

- Figure S1 Dynamics of the fusion peptide of SARS-CoV-2 Spike protein.

- Figure S2 Pharmacophore model used for virtual screening.

- Figure S3 Activities of R enantiomer of $\mathrm{C} 2$ and predicted binding model of $\mathrm{R}$ and $\mathrm{S}$.

- Figure S4 Predicted binding model of $\mathrm{C} 1$ analogs in the SARS-CoV-2 S FP pocket.

- Figure S5 Predicted binding model of $\mathrm{C} 2$ analogs in the SARS-CoV-2 S FP pocket.

- Figure S6 Activities of $\mathrm{C} 2$ analogs in the PP entry against SARS-CoV-2, SARS-CoV-1 and MERS

- Figure S7 Predicted binding model of fendiline and vortioxetine.

- Table S1 Physicochemical properties and commercial source of identified inhibitors of SARS-CoV-2.

- Experimental Methods 
A

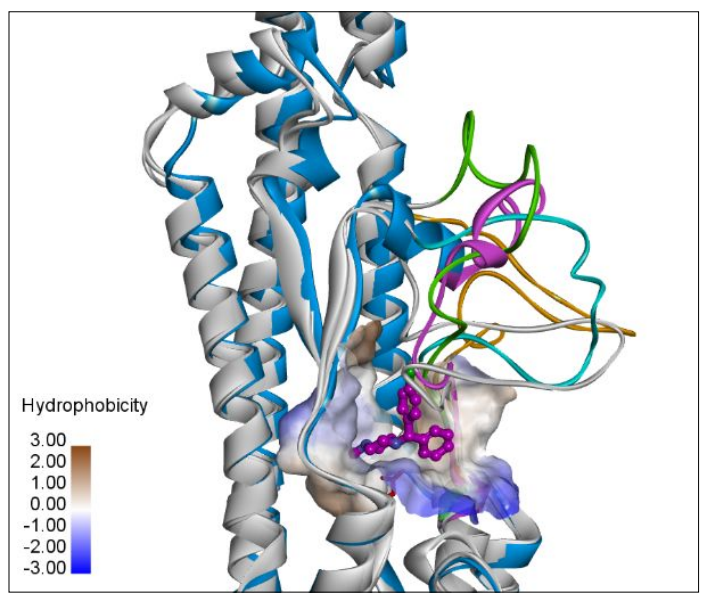

B

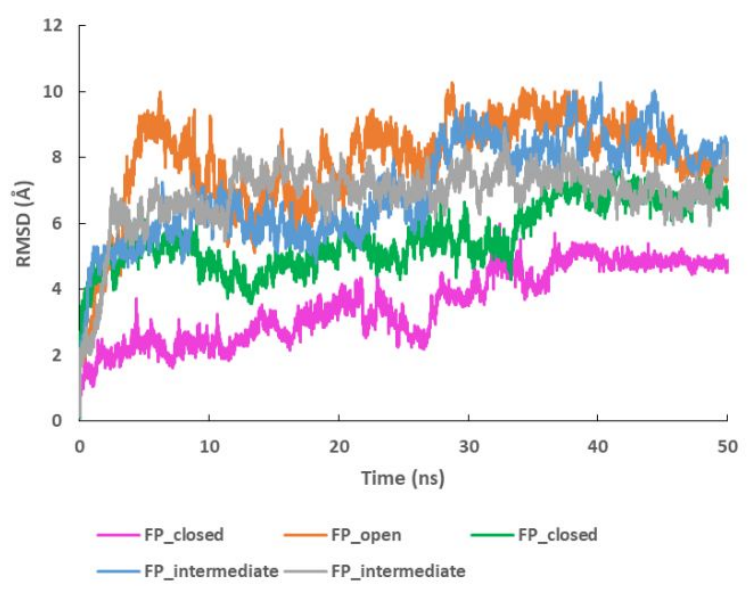

Figure S1. Dynamic property of the fusion peptide of SARS-CoV-2 Spike protein. A) Superimposed structural models of the FP in different conformational state. The closed conformation observed in the trimeric complex of spike protein (PDB 6XR8) is shown in blue and the structured FP loop is shown in magenta. The FP-disordered conformation of S structure (PDB $6 \mathrm{VSB}$ ) is showed in grey and the re-modeled FP in the closed, intermediate, and open conformations are shown in different color. The FP models were generated using Loop Modeler in MOE program. The FP binding pocket is rendered in hydrophobic surface and small molecule CCZ docked in the pocket is shown in sticks and balls. B) The RMSD plot of FP in the 50-ns MD simulations. 


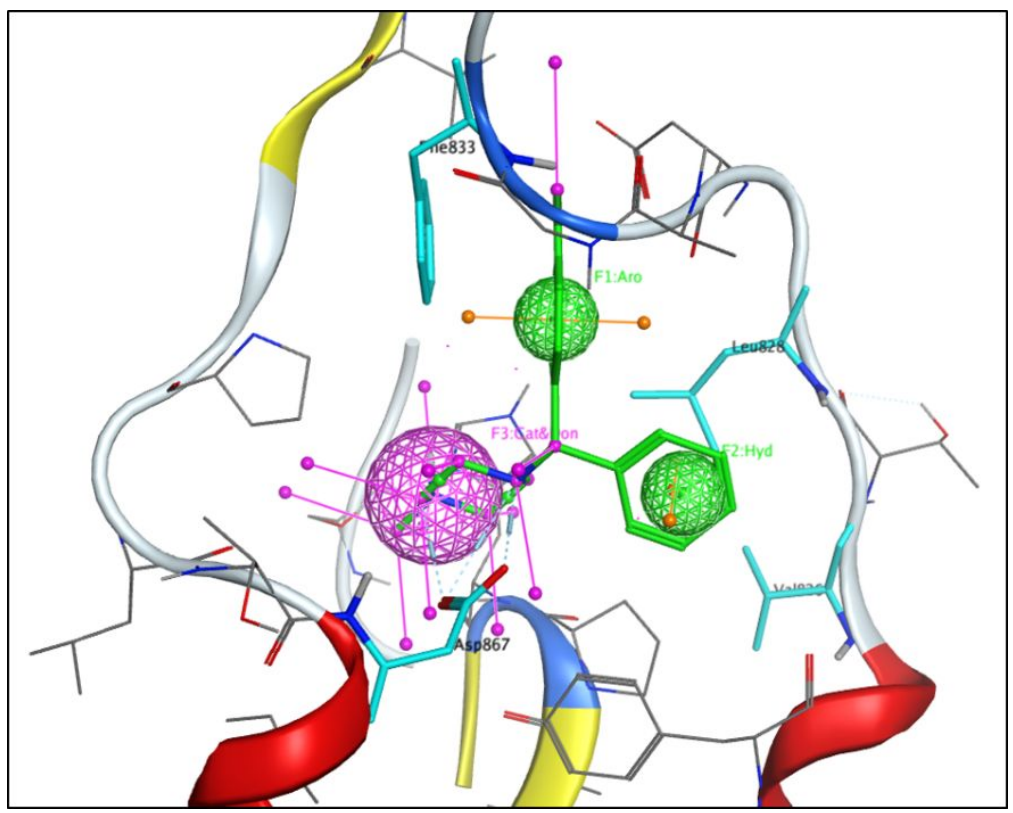

Figure S2. Pharmacophore model used for virtual screening. The pharmacophore models were generated based on the predicted binding interaction of CCZ at the FP binding site using MOE. Three pharmacophoric features were included: 1) an Don2 projected H-bond donor feature placed on the sidechain of Asp867; 2) an Aro centroids feature matching aromatic interaction with Phe833; 3) an Hyd centroids feature matching hydrophobic interactions with Leu828 and Val826. 
A
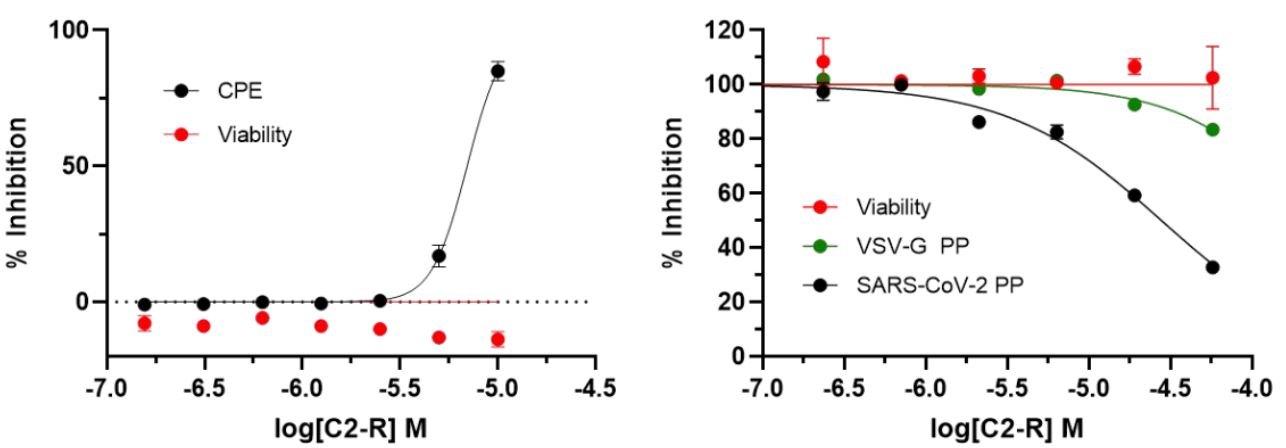

B

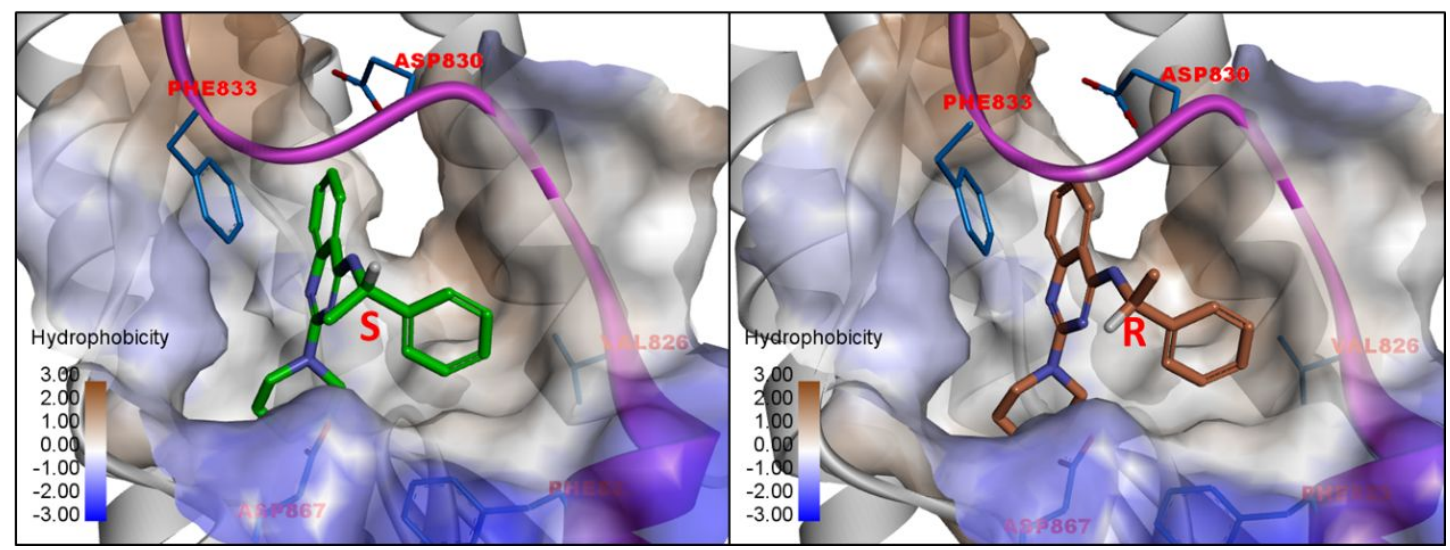

Figure S3. A) Activities of R enantiomer of C2 in the CPE and PP assay. B) Predicted binding model of $\mathrm{S}$ and $\mathrm{R}$ enantiomers of $\mathrm{C} 2$. The chiral center of $\mathrm{C}$ atom is labeled. The binding pocket is rendered in hydrophobic surface and the FP is colored in magenta. Key interacting residues in the binding pocket are shown in sticks. The methyl group at the chiral center pointed out to the solvent-exposed region. Consistent with experimental results of similar activity in the CPE and PP assays, the predicted binding energies of $\mathrm{S}$ and $\mathrm{R}$ enantiomers were -6.43 and $-6.29 \mathrm{kcal} / \mathrm{mole}$, respectively. 


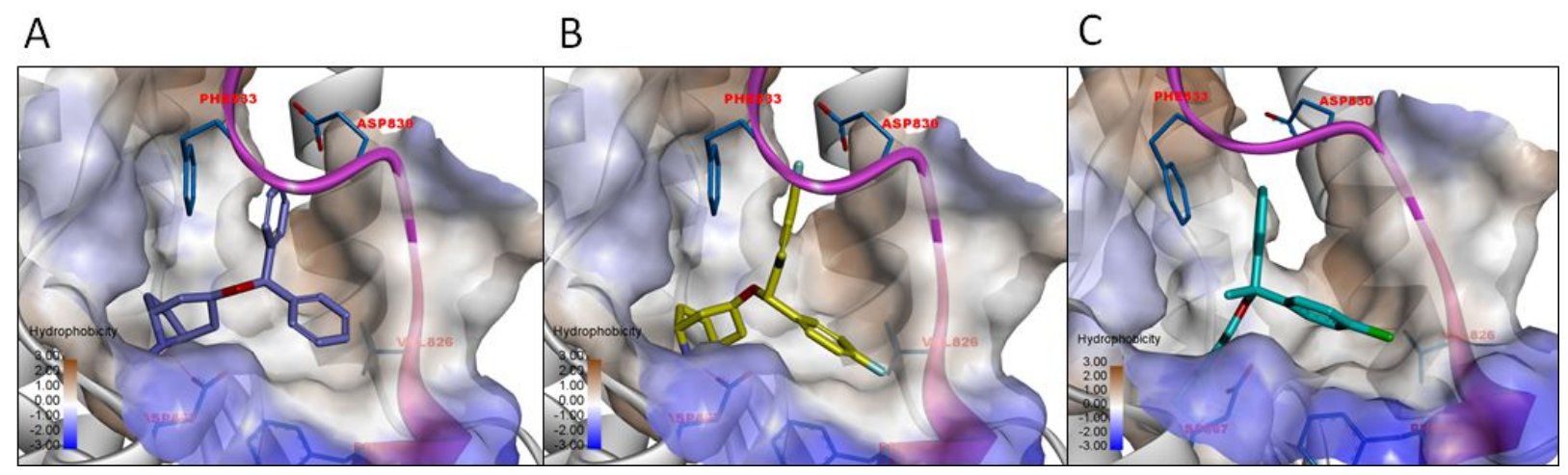

Figure S4. Predicted binding model of (A) benztropine, (B) difluorobenztropine, and (C) clemastine in the FP pocket of SARS-CoV-2 spike protein. The binding pocket is rendered in hydrophobic surface and the FP is colored in magenta. Key interacting residues in the binding pocket are shown in sticks. 


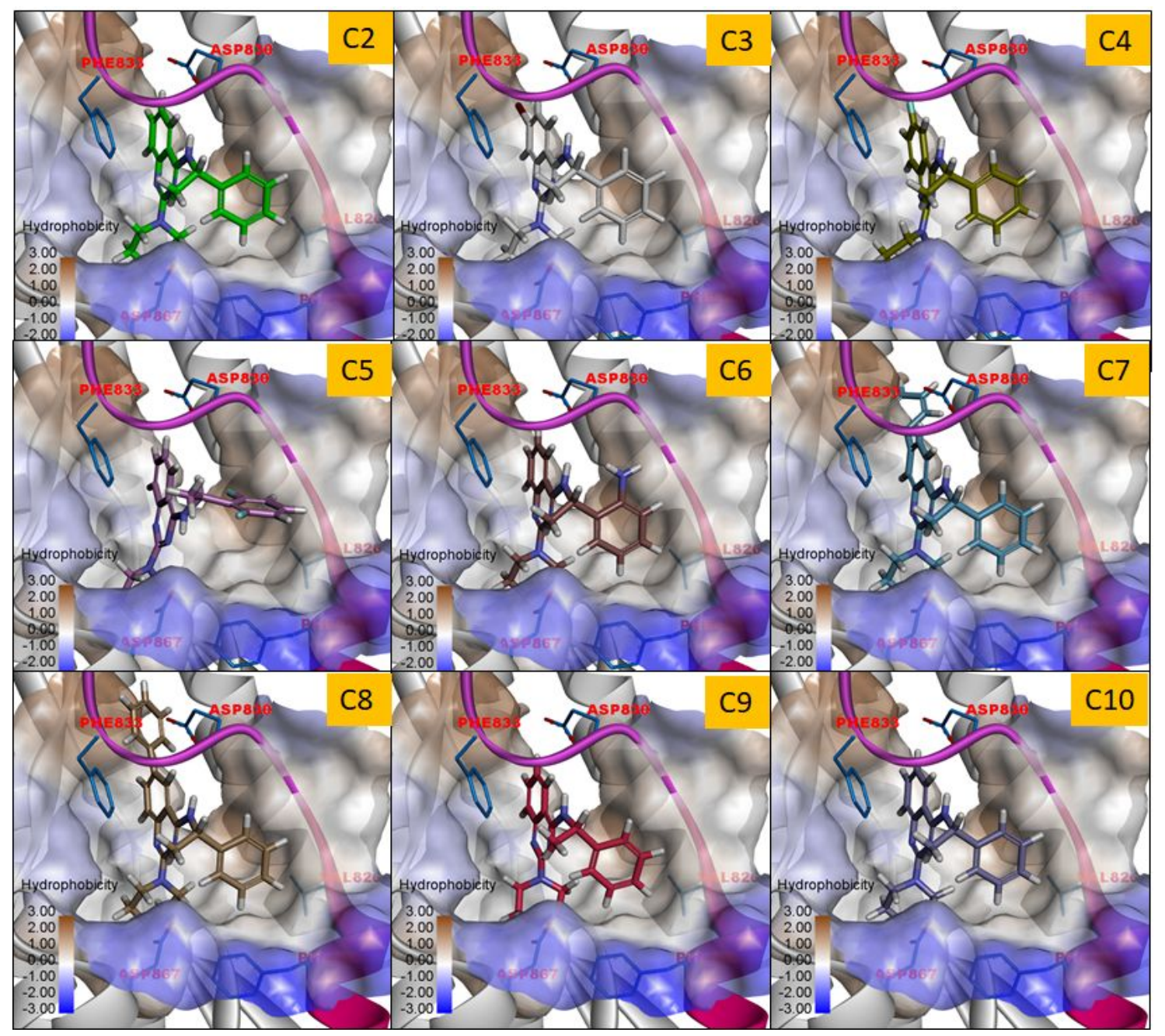

Figure S5. Predicted binding model of $\mathrm{C} 2$ analogs in the FP pocket of SARS-CoV-2 spike protein. The binding pocket is rendered in hydrophobic surface and the FP is colored in magenta. Key interacting residues in the binding pocket are shown in sticks. 
C6
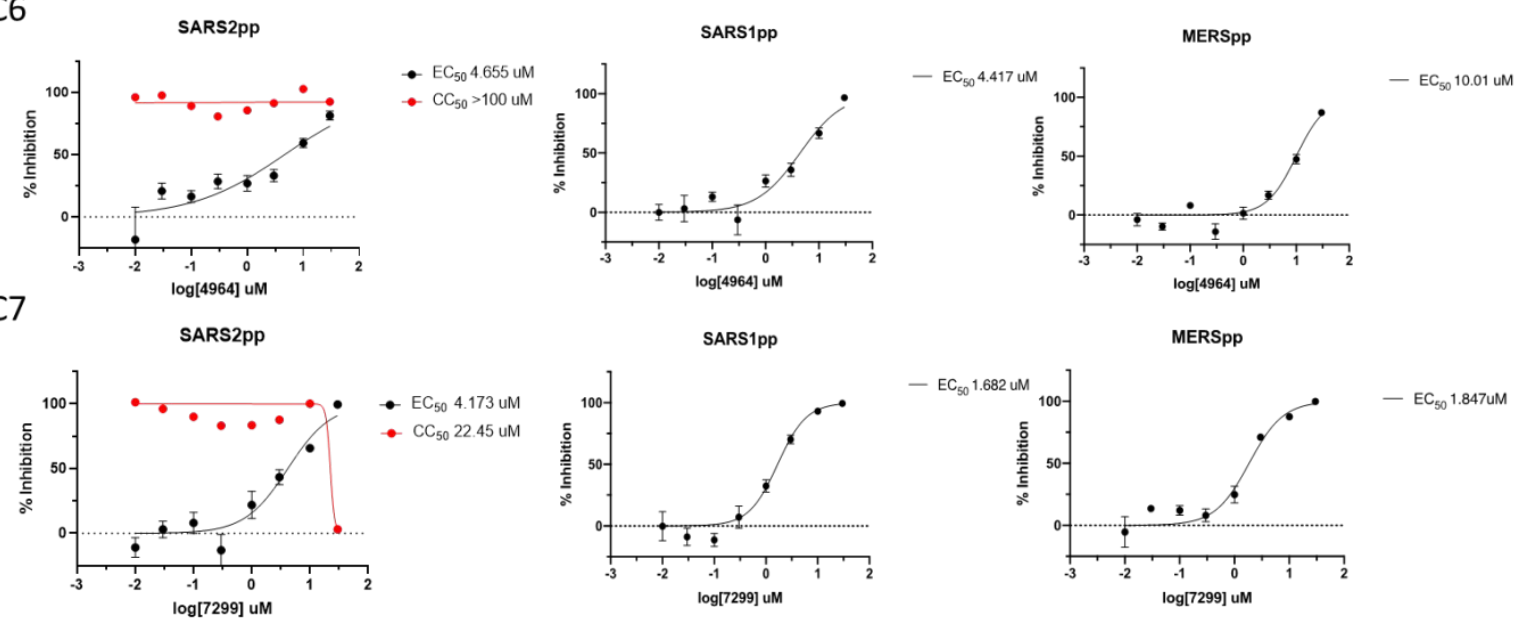

C8

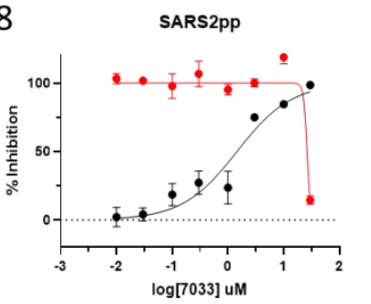

- $\mathrm{EC}_{50} 1.452 \mathrm{um}$
- $\mathrm{CC} 5026.32 \mathrm{uM}$

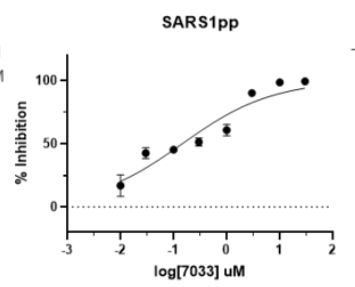

- $\mathrm{EC}_{50} 0.1461 \mathrm{um}$

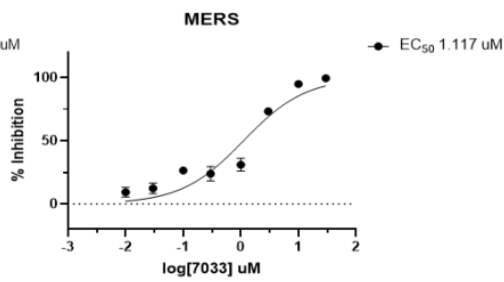

C9
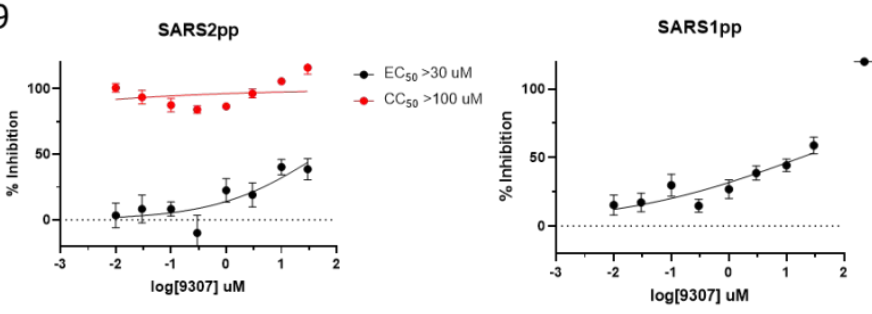

- $\mathrm{EC}_{50} 17.95 \mathrm{u}$

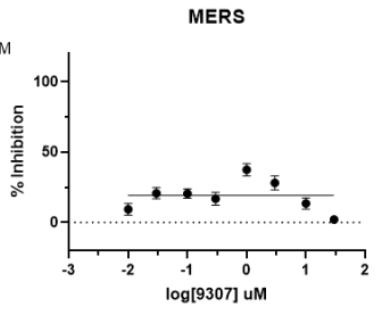

C10
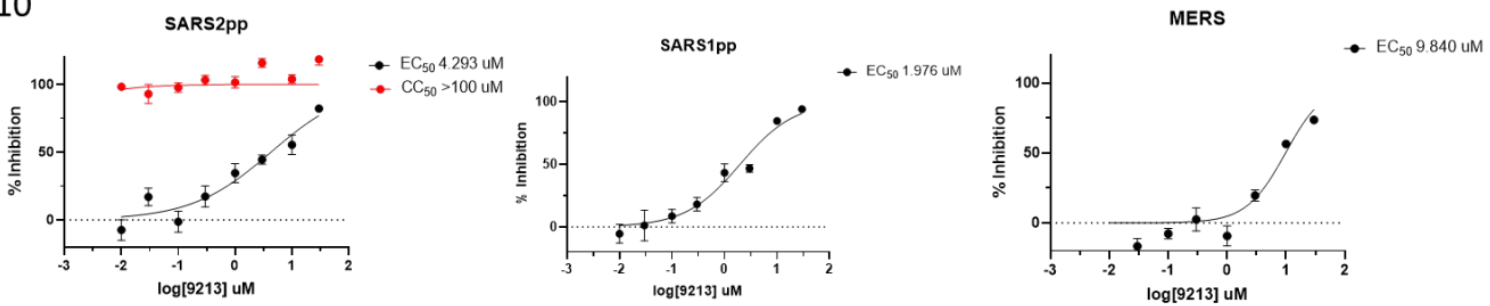

Figure S6. Activities of $\mathrm{C} 2$ analogs in the PP entry against SARS-CoV-2, SARS-CoV-1 and MERS. 

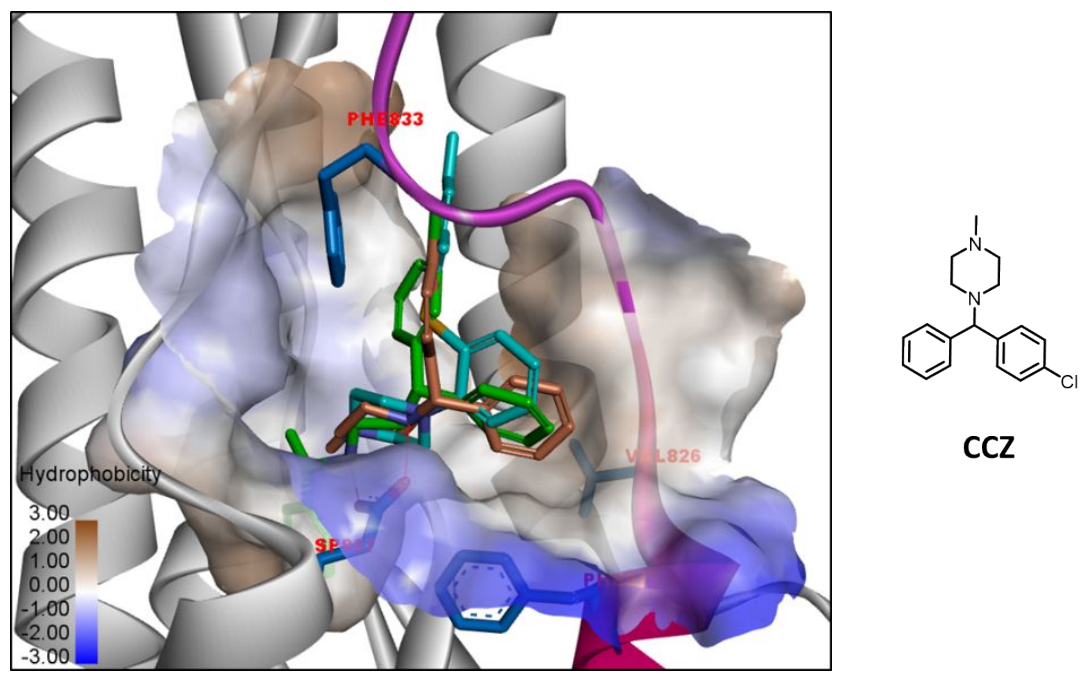<smiles>CC(NCCC(c1ccccc1)c1ccccc1)c1ccccc1</smiles><smiles>Cc1ccc(Sc2ccccc2N2CCNCC2)c(C)c1</smiles>

Fendiline

Vortioxetineat

Figure S7. Predicted binding model of CCZ (brown), fendiline (green) and vortioxetine (cyan) in the FP pocket of SARS-CoV-2 spike protein. The binding pocket is rendered in hydrophobic surface and the FP is colored in magenta. Key interacting residues in the binding pocket are shown in sticks. 


\section{Supplementary Table}

Table S1. Physicochemical properties and commercial source of identified inhibitors of SARSCoV-2.

\begin{tabular}{|c|c|c|c|c|c|c|c|c|c|c|}
\hline Name & MW & $\begin{array}{l}\text { HB- } \\
\text { Acc }\end{array}$ & $\begin{array}{l}\text { HB- } \\
\text { Don }\end{array}$ & $\begin{array}{l}\text { Rot. } \\
\text { Bond }\end{array}$ & $\begin{array}{l}\text { TPS } \\
\mathrm{A} \\
\end{array}$ & NCGC ID & $\begin{array}{l}\text { PubChem } \\
\text { CID }\end{array}$ & Supplier & $\begin{array}{l}\text { Supplier } \\
\text { ID }\end{array}$ & $\begin{array}{l}\text { Compound } \\
\text { Purity* }\end{array}$ \\
\hline Clobenztropine & 341.9 & 2 & 0 & 4 & 12.5 & NCGC00015195 & 1687 & Tocris & 917 & $>=99 \%$ \\
\hline Benztropine & 307.4 & 2 & 0 & 4 & 12.5 & NCGC00013495 & 2344 & APExBIO & B1554 & $>=99 \%$ \\
\hline Difluorobenztropine & 343.4 & 4 & 0 & 4 & 12.5 & NCGC00024872 & 3929516 & Tocris & 918 & $>=99 \%$ \\
\hline Clemastine & 343.9 & 2 & 0 & 6 & 12.5 & NCGC00016710 & 26987 & Microsource & 1500191 & $>=99 \%$ \\
\hline D3- $\beta$ Arr & 333.4 & 5 & 2 & 4 & 53.1 & NCGC00379308 & 2950121 & MCE & HY-124867 & $>=99 \%$ \\
\hline
\end{tabular}

*Compound purity was measured by LC-MS. 


\section{Experimental Methods}

\section{Molecular modeling and docking}

Modeling and docking studies of small molecule inhibitors to the FP binding pocket of SARSCoV-2 were performed using the Molecular Operating Environment (MOE) program. ${ }^{1}$ The disordered FP loop (residues 829-852) in the structure of RBD-up spike protein of (PDB 6VSB) was re-modeled using the Loop Modeler in MOE. A total of 500 conformers of the loop were generated and clustered. Five representatives of open and closed conformations from major clusters were selected.

Ensemble docking was performed using MOE Dock in MOE and AutoDock Vina. ${ }^{2}$ The closed conformation of FP in the RBD-down Spike protein (PDB 6XR8) was also used in ensemble docking. Prior to docking, the 3D structures were prepared using the Structure Preparation module in MOE. The ligand induced fit docking protocol in MOE Dock was applied and binding affinity was evaluated using the GBVI/WSA score. The default parameters in AutoDock Vina were used with a grid box centered on residue Asp867. The size of grid box was defined by 25 x 25 x $25 \AA$ to encompass the entire FP binding site. The top-ranked 10 poses from MOE Dock and AutoDock Vina were retained and visually inspected.

MD simulations of the FP structural models in the open and closed forms and inhibitor-bound complexes were conducted using the AMBER18 package. ${ }^{3}$ To reduce the complexity, only the S2 subunit of the spike protein monomer was employed in the MD simulations. The solvated protein systems were subjected to a thorough energy minimization prior to MD simulations by first minimizing the water molecules while holding the solute frozen (1000 steps using the steepest descent algorithm), followed by 5,000 steps of conjugate gradient minimization of the whole system to relax the system. The simulated system in explicit solvate was first subjected to a gradual temperature increase from $0 \mathrm{~K}$ to $300 \mathrm{~K}$ over $100 \mathrm{ps}$, and then equilibrated for $500 \mathrm{ps}$ at $300 \mathrm{~K}$, followed by a production run of $50 \mathrm{~ns}$. Trajectory analysis and were performed using the cpptraj module in the AmberTools $18 .^{3}$

\section{Pharmacophore-based virtual screening}

The pharmacophore models were generated based on the predicted binding interaction of CCZ at the FP binding site using MOE. Three pharmacophoric features were included: 1) an Don2 
projected H-bond donor feature placed on the sidechain of Asp867; 2) an Aro centroids feature matching aromatic interaction with Phe833; 3) an Hyd centroids feature matching hydrophobic interactions with Leu828 and Val826. To enrich the structural diversity of potential hits, the 3D shape-based searching was also applied using ROCS. ${ }^{4} \mathrm{~A}$ total of 10,000 hits were extracted from the pharmacophore and 3D shape-based searching, followed by ensemble docking to the FP binding site of SRAS-CoV-2 spike protein using AutoDock Vina with default parameters. The top-ranked 2000 compounds from each docking were retained for analysis. Structural clustering was performed using MOE. All compounds from each cluster and singletons were visually inspected. Finally, 120 compounds were selected based on: 1) structural representatives of each cluster, 2) predicted binding energy, 3) H-bonding interaction with Asp867, 4) promiscuous compounds with potential undesirable functionalities and PAINS alert were generally discarded.

\section{SARS-CoV-2 cytopathic effect (CPE) assay}

SARS-CoV-2 CPE assay was conducted at Southern Research Institute (Birmingham, AL) as described in previous reports. ${ }^{5}$ In brief, high ACE2 expressing Vero E6 cells were inoculated with SARS-CoV-2 (USA_WA1/2020) at 0.002 M.O.I. After infection of $72 \mathrm{~h}$ at $37{ }^{\circ} \mathrm{C}$ and $5 \% \mathrm{CO}_{2}$,

the cell viability was examined with CellTiter-Glo ATP content assay kit (Promega, Madison, WI, USA). CPE raw data were normalized to noninfected cells and virus infected cells only which were set as $100 \%$ efficacy and 0 efficacy, respectively. In addition, the compound cytotoxicity was evaluated in the same cells by measuring ATP content in the absence of virus. Compound cytotoxicity raw data were normalized with wells containing cells only as $100 \%$ viability $(0 \%$ cytotoxicity), and wells containing media only as $0 \%$ viability ( $100 \%$ cytotoxicity).

\section{SARS-CoV-2-S pseudotyped particle entry assay}

SARS-CoV-2 Spike pseudotyped particle (PP) entry assay was performed as previously described. ${ }^{6}$ Briefly, 3500 ACE2-GFP cells were seeded in $15 \mu \mathrm{L}$ of media in 384-well plates and incubated at $37{ }^{\circ} \mathrm{C}$ and $5 \% \mathrm{CO} 2$ overnight. Cells were treated with compound using acoustic dispensing. Fifteen microliters of PPs was added, and plates were spin-inoculated at $1500 \mathrm{rpm}$ (453g) for $45 \mathrm{~min}$ and incubated for $48 \mathrm{~h}$ at $37{ }^{\circ} \mathrm{C}$ and $5 \% \mathrm{CO} 2$. The supernatant was removed, and $20 \mu \mathrm{L} /$ well of Bright-Glo (Promega) was added; the mixture was incubated for $5 \mathrm{~min}$ at room temperature. The luminescence signal was measured using a PHERAStar plate reader (BMG 
Labtech). All data were normalized to DMSO and SARS-CoV-2 PP treated wells as 0\% efficacy and DMSO and delEnv PP treated wells as 100\% efficacy. The cytotoxicity of the compounds was measured in mock PP treated plates using ATPlite reagent (PerkinElmer). Data was normalized to DMSO treated cells as $100 \%$ cell viability and DMSO treated media as $0 \%$ cell viability.

\section{Antiviral assays with a cell-based infection system of pseudotyped viral particles}

Pseudotyped viral particles enveloped by SARS-CoV, SARS-CoV-2, or MERSCoV Spike proteins (S-pp) were generated in 293T cells as described in the literature. Briefly, the 293 T cells were transfected with the spike protein expression plasmids corresponding to each respective coronavirus using FuGENE® 6 Transfection Reagent (Promega). 24 h later, the cells were infected with a replication-defective recombinant vesicular stomatitis virus (VSV) pseudotyped with the native VSV-G glycoprotein (VSV-Gpp), in which the firefly luciferase reporter gene was inserted in place of the VSV-G sequence. $4 \mathrm{~h}$ later, uninfected VSVGpp were washed out and newly produced SARS-CoV-Spp, SARS-CoV-2-Spp, or MERS-CoVSpp were harvested at $24 \mathrm{~h}$ and $48 \mathrm{~h}$ following infection. The viruses were titrated based on the luciferase activity by reinfecting the viruses in the naïve cells and stored at $-80^{\circ} \mathrm{C}$ freezers.

The antiviral activity was tested against the pseudotyped viruses in a hepatoma cell line Huh7 cells. First, Huh7 cells were seeded in white 96-well plates (Greiner BioOne, Kremsmunster, Austria) at a density of 14,000 cells per well for overnight. The cells were treated with various compounds at $1 / 2 \log$ dose intervals as indicated in the figures, immediately followed by the addition of pseudotyped virus with a targeted reading of about 200,000 relative luciferase activity. Luciferase assay was performed $24 \mathrm{~h}$ post-infection using the Amplite ${ }^{\mathrm{TM}}$ Luciferase Reporter Gene Assay Kit (AAT Bioquest, Sunnyvale, CA, USA) according to manufacturer's instructions and analyzed using a POLARstar Omega plate reader (BMG LABTECH, Ortenberg, Germany). A parallel plate identically treated as the infection assay plate was used to determine the cytotoxicity of various compounds. Cytotoxicity assay was performed using The PhosphoWorks ${ }^{\text {TM }}$ Luminometric ATP Assay Kit (AAT Bioquest).

\section{LC/MS analysis}

Compound purity was assessed using LC/MS. The analysis was performed on a Agilent LC/MS. A 6.8 minute gradient of 4 to $100 \%$ acetonitrile (containing $0.025 \%$ trifluoroacetic acid) 
in water (containing $0.05 \%$ trifluoroacetic acid) was used with a 8.5 minute run time at a flow rate of $0.8 \mathrm{~mL} / \mathrm{min}$. The column was an Agilent Eclipse XDB-C18, 3.5 micron, 3.0

$\mathrm{x} 75 \mathrm{~mm}$. Purity determination was performed using a Diode Array Detector at 220nM and Evaporative Light Scattering Detector as backup. Mass Determination is performed using a Agilent 6125B mass spectrometer. Data was analyzed using the Agilent Masshunter software.

\section{References}

1. Molecular Operating Environment (MOE) 2019.01. Chemical Computing Group ULC, Montreal, QC, Canada.

2. Trott, O.; Olson, A. J., AutoDock Vina: improving the speed and accuracy of docking with a new scoring function, efficient optimization, and multithreading. J Comput Chem 2010, 31 (2), 455-61.

3. $\quad$ Case, D. A.; Ben-Shalom, I. Y.; Brozell, S. R.; D.S., C.; T.E., C. I.; Cruzeiro, V. W. D.; Darden, T. A.; Duke, R. E.; Ghoreishi, D.; Simmerling CL; Wang J; Luo R; Merz KM; Wang B; Pearlman DA; Crowley M; Tsui V; Gohlke H; Mongan J; Hornak V; Cui G; Beroza P; Schafmeister C; Walker, R. C.; Wei, H.; Wolf, R. M.; Wu, X.; Xiao, L.; York, D. M.; Kollman, P. A., AMBER18. University of California, San Francisco: 2018.

4. Hawkins, P. C.; Skillman, A. G.; Nicholls, A., Comparison of shape-matching and docking as virtual screening tools. J Med Chem 2007, 50 (1), 74-82.

5. $\quad$ Chen, C. Z.; Shinn, P.; Itkin, Z.; Eastman, R. T.; Bostwick, R.; Rasmussen, L.; Huang, R.; Shen, M.; Hu, X.; Wilson, K. M.; Brooks, B. M.; Guo, H.; Zhao, T.; KlumpThomas, C.; Simeonov, A.; Michael, S. G.; Lo, D. C.; Hall, M. D.; Zheng, W., Drug Repurposing Screen for Compounds Inhibiting the Cytopathic Effect of SARS-CoV-2. Front Pharmacol 2020, 11, 592737.

6. $\quad$ Chen, C. Z.; Xu, M.; Pradhan, M.; Gorshkov, K.; Petersen, J. D.; Straus, M. R.; Zhu, W.; Shinn, P.; Guo, H.; Shen, M.; Klumpp-Thomas, C.; Michael, S. G.; Zimmerberg, J.; Zheng, W.; Whittaker, G. R., Identifying SARS-CoV-2 Entry Inhibitors through Drug Repurposing Screens of SARS-S and MERS-S Pseudotyped Particles. ACS Pharmacol Transl Sci 2020, 3 (6), 1165-1175. 